\title{
Effects of Flaxseed oil supplementation on renal dysfunction due to ischemia/reperfusion in rat
}

Tahmineh Mokhtari ${ }^{1,2}$, Hedyeh Faghir Ghanesefat ${ }^{3}$, Gholamreza Hassanzadeh ${ }^{1}$, Ardeshir Moayeri $^{4}$, Seyed Mohammad Jafar Haeri ${ }^{5}$, Alireza Rezaee Kanavee ${ }^{2}$, Seyyed Majid Mousavi Movahed $^{2 *}$

1. Department of Anatomy, School of Medicine, Tehran University of Medical Sciences, Tehran, Iran

2. Baharloo Hospital, Tehran University of Medical Sciences, Tehran, Iran

3. Department of Pharmacology, School of Medicine, Tehran University of Medical Sciences, Tehran, Iran

4. Department of Anatomy, School of Medicine, Ilam University of Medical Sciences, Ilam, Iran

5. Biology and Anatomical Sciences, School of Medicine, Shahid Beheshti University of Medical Sciences, Tehran, Iran

*Corresponding author:Tel: +98 9121535223 Fax: +98 2155648189

Address: Baharloo Hospital, Tehran University of Medical Sciences, Tehran, Iran

E-mail: moosavimovahed@gmail.com

Received; 2016/05/20 revised; 2016/06/4 accepted; 2016/07/21

\section{Abstract}

Introduction: This study was designed to evaluate the effects of treatment with flaxseed oil (FSO) on renal ischemia-reperfusion (RIR) injuries in rats.

Materials and methods: In this study, 32 Wistar rats were randomly studied in four groups: $\mathrm{Co}+\mathrm{NS}$ (Control group with normal saline administration), $\mathrm{Sh}+\mathrm{NS}$ (sham group with normal saline administration), RIR+NS and RIR+FSO. FSO $(0.2 \mathrm{ml})$ was administered orally (gavage) for 14 days $(\sim 800 \mathrm{mg} / \mathrm{kg}$ body weight). Blood samples were collected for the detection of blood urea nitrogen (BUN) and creatinine levels. Malondialdehyde (MDA) and superoxide dismutase (SOD) levels were evaluated in the renal tissue. Tubular damages were examined using histopathological studies.

Results: Significantly elevated MDA $(\mathrm{P}<0.05)$ and depressed SOD levels $(\mathrm{P}<0.05)$ Comparison between RIR+NS group and Control+NS and Sh+NS groups revealed in the condition of RIR. Treatment with FSO, however, significantly lowered the MDA $(\mathrm{P}<0.05)$ and enhanced SOD levels $(\mathrm{P}<0.05)$ after RIR injury. Histopathological results confirmed the biochemical studies and tubular necrosis score was reduced in the RIR+FSO group.

Conclusion: This study therefore suggests that the aqueous flaxseed oil may be useful agents for the prevention of renal ischemia-reperfusion (RIR)-induced oxidative injury in rats.

Keywords: Flaxseed oil, Renal ischemia-reperfusion, Oxidative injury, MDA, SOD

\section{Introduction}

Renal Ischemia reperfusion (RIR) injury is a common complication during a number of clinically important conditions, including hydronephrosis and sepsis, hypertension or surgical procedures such as cardiac bypass, kidney transplantation, as well as partial nephrectomy leading $(1,2)$.Subsequent clinical outcomes of RIR present as acute renal failure (ARFs) with wide range of impairment from minor alterations to major functional failures with requirement for dialysis or kidney transplantation (3). Moreover, ischemia followed by re-flow the blood supply (reperfusion) can lead to additional renal tissue damages (4). In this condition, the 
development of oxidative stress via generation of reactive oxygen species (ROS) leads to acute renal failure (ARF) and renal dysfunction due to ischemia reperfusion (IR) $(5,6)$.

Antioxidant enzymes such as catalase (CAT), glutathione peroxidase (GPx), and superoxide dismutase (SOD) contribute to remove ROSs from involved tissues. Generation of ROSs and/or deactivation of antioxidant enzymes such as SOD via nitration of their structures can promote the oxidative stress in RIR injury which induces extensive impairments (7).

Furthermore, lipid peroxidation due to oxidative stress is a catalytic mechanism leading to oxidative destruction of cellular membranes in RIR injury. Malondialdehyde (MDA) is an important factor to record the rate of lipid peroxidation (8). So, targeting the oxidative stress by ROS scavengers can protect against IR injuries (9).

Using the natural dietary nutrients with antioxidant and/or scavenging characteristics is beneficial to control the oxidative stress conditions (10). Flaxseed (FS, Linum usitatissimum) as a nutritional whole grain and FS oil (FSO) are considered as a dietary nutrient for countries in Asia, Africa and Europe. This nutrient has been introduced as a richest plant based source of omega-3 polyunsaturated fatty acids and lignans $(11,12)$. The ROSs scavenging characteristics of FS lignans were demonstrated in-vitro by either inhibiting lipid peroxidation or direct hydroxyl radical scavenging activities (13).

Accordingly, this study was designed to study detailed pathological and biochemical events of ARFs due to RIR injury and its possible mitigation by FSO. It was hypothesized that FSO would reduce IR-induced adverse effects on kidney through its antioxidant characteristics.

\section{Materials and methods}

Experimental animals: Thirty-two healthy male Wistar rats approximately ranging in weight from $280 \mathrm{~g}$ to $350 \mathrm{~g}$, obtained from the animal house of the Pharmacology department, Tehran University of Medical sciences, Tehran, Iran, were used in the present study. Animals were kept in cages in a room under standard condition of relative humidity $(55 \pm 5 \%)$ and temperature $(25 \pm$ $2^{\circ} \mathrm{C}$ ) with a 12-hr light/dark cycle, and at $25 \pm 2^{\circ} \mathrm{C}$ room, according to local ethical guidelines of Tehran University of Medical Science, Tehran, Iran.

Renal ischemia reperfusion (IR) Surgery: Animal was anesthetized with intraperitoneal injection of ketamine hydrochloride $(50 \mathrm{mg} / \mathrm{kg})+$ xylazine $(10$ $\mathrm{mg} / \mathrm{kg}$ ) and placed at right flunk position. The right nephrectomy was performed after a minimal dissection under last rib. Before the animals were subjected to IR injury, they were allowed to recover for one week. On the 7th day following operation, Animals were fasted overnight and under anesthesia, using minimal dissection under the left rib, the pedicle of left kidney was isolated. The renal pedicle was occluded for $45 \mathrm{~min}$ and subjected to reperfusion for $24 \mathrm{hr}$ to induce IR. Then, the incision was sutured and rats were allowed to recover with free access to water and food.

Experimental design: Rats were randomly allocated to four groups $(n=8$ for each group) as follow:

Group I: Co+NS. Served as vehicle control and received normal saline $(0.2 \mathrm{ml}$ saline/rat) by oral administration (gavage) for 14 days.

Group II: Sh+NS. Sham group subjected to surgery without ischemia induction and received saline $(0.2 \mathrm{ml}$ saline/rat) by oral administration (gavage) for 14 days .

Group III: RIR+NS. Induced renal ischemia and received saline by oral administration for 14 days. 
Group IV: RIR+FSO. Induced renal ischemia and received $0.2 \mathrm{ml}$ of flaxseed oil by gavage (orally) once daily for 14 days $(\sim 800 \mathrm{mg} / \mathrm{kg}$ body weight $)$.

Serum parameters: After 14 days, rats were sacrificed and their blood samples were withdrawn and centrifuged at $4000 \mathrm{~g}$ for $10 \mathrm{~min}$ at $4^{\circ} \mathrm{C}$ to separate serum and stored at $-80{ }^{\circ} \mathrm{C}$ to determine the biochemical levels. The blood urea nitrogen (BUN) and creatinine levels in the serum were determined to assess the renal function.

Preparation of homogenates and Assay of enzymes: The left kidney was divided into halves. The kidney cortex in one part was carefully separated from medulla according to method was described by Khundmiri et al. (2005) (14). After frizzing in the liquid nitrogen, the cortex was stored at $-80^{\circ} \mathrm{C}$ to use in the next biochemical assessments.

To assay of enzymes involved in free radical scavenging, the cortex was homogenized immediately in $0.1 \mathrm{M}$ Tris$\mathrm{HCl}$ buffer, $\mathrm{pH} 7.5$, using Potter-Elvehjem homogenizer (Remi Motors, Mumbai, India) and a $15 \%(\mathrm{w} / \mathrm{v})$ homogenate was prepared. To remove cell debris and the supernatant, it was centrifuged at $3000 \times \mathrm{g}$ at $4 \mathrm{C}$ for $15 \mathrm{~min}$ and stored at $20 \mathrm{C}$ for assaying free-radical scavenging enzymes. The supernatant $(10 \%)$ was used for the various biochemical determinations. Afterwards, renal Malondialdehyde (MDA) levels as an index for lipid peroxidation and superoxide dismutase (SOD) levels as an endogenous antioxidant enzyme were measured. Tissue MDA levels were determined using the thiobarbituric acid reactive substances (TBARS) method (15) and SOD level was measured by a spectrophotometric method of Misra et al (16).

Histopathology: The other part was fixed in formalin $10 \%$ for histo-pathological analysis. It was dehydrated through a graded-alcohol series. Then, the transverse kidney slices were embedded in paraffin and sectioned at $5 \mu \mathrm{m}$ slides. After providing slides stained with hematoxylin eosin (H\&E), the ischemic tubular damage was assessed using a modified form of grading scales (18). The numerical score (0 to 4) was described as follows: (0) no damage; (1) unicellular patchy isolated necrosis; (2) <25\% tubular necrosis (3), tubular necrosis 25-50\%; and (4) tubular necrosis $>50 \%$.

\section{Statistical analysis}

All quantitative variables were presented as mean \pm standard error of mean (SEE). For comparing the data in the groups, the one-way ANOVA analysis with post hoc of Tukey test was used. The comparison of total histopathological scores between the groups was made by nonparametric Kroskal-Wallis tests. All statistical analyses were performed using the SPSS software (Statistical Package for the Social Sciences, version 22, SPSS Inc, Chicago, Ill, USA). A P-value less than 0.05 was taken to indicate significance.

\section{Results}

Effects of FSO on serum variables in rats with renal IR: Table 1 shows that there were significant differences between the studied groups $(\mathrm{P}<0.05$, Table 1$)$. These significant differences were related to increase in the serum creatinine and BUN concentrations in IRI+NS, IRI+FSO groups compared with their corresponding values in the Co+NS and $\mathrm{Sh}+\mathrm{NS}$ groups $(\mathrm{P}<0.05$, Table 1). Serum creatinine and BUN levels in the IRI+FSO significantly decreased in comparison with the IRI+NS group $(\mathrm{P}<0.05$, Table 1$)$.

Effects of FSO on kidney anti-oxidant parameters: According to Table 2, significant differences were observed in the SOD and MDA levels of kidney tissue. There were no significant differences between $\mathrm{Co}+\mathrm{NS}$ and $\mathrm{Sh}+\mathrm{NS}$ groups $(\mathrm{P}<0.05$, Table 2). In IRI+NS and IRI+FSO groups, a significant decrease in the levels of enzymatic anti-oxidants namely SOD and a significant increase in 
the lipid peroxide (MDA) levels were recorded compared with $\mathrm{Co}+\mathrm{NS}$ and Sh+NS Groups indicating the oxidative stress $(\mathrm{P}<0.05$, Table 1). Furthermore, there was a significant difference between the IRI+NS and IRI+FSO groups in the SOD and MDA concentrations $(\mathrm{P}<0.05$, Table 1).

Table 1: Effect of FSO on Serum Variables in rats with renal IR.

\begin{tabular}{lcc}
\hline Treatment groups & BUN & Cr \\
& $(\mathbf{m g} / \mathbf{d l})$ & $8.65 \pm 0.27$ \\
Co+NS & $6.57 \pm 0.32$ & $9.21 \pm 0.22$ \\
Sh+NS & $7.1 \pm 0.31$ & $23.17 \pm 0.29 * \#$ \\
RIR+NS & $16.32 \pm 0.36^{* \#}$ & $15 \pm 0.25^{*}+\dagger$ \\
RIR+FSO & $10.12 \pm 0.29 * \# \dagger$ & \\
\hline
\end{tabular}

All values are demonstrated as Mean \pm SEE. ${ }^{*} \mathrm{p}<0.05$ compared to Co+NS; ${ }^{\#} \mathrm{p}<0.05$ compared to $\mathrm{Sh}+\mathrm{NS} . \dagger$ $\mathrm{p}<0.05$ compared to RIR+NS. BUN: Blood urea nitrogen, Cr: Creatinine, Co+NS: normal group without ischemia and with oral Normal saline (NS), Sh+NS: sham group subjected to surgery procedure without ischemia induction and with oral NS; IRI+NS: Ischemia induction group with oral NS, IRI+FSO: Ischemia induction group with oral FSO.

Table 2. Effect of FSO on antioxidant parameters of renal tissue.

\begin{tabular}{lcc}
\hline Treatment groups & SOD & MDA \\
& $(\mathbf{U} / \mathbf{g})$ & $10.21 \pm 1.27$ \\
Co+NS & $821.21 \pm 12.27$ & $11.13 \pm 1.66$ \\
Sh+NS & $810.55 \pm 12.98$ & $42.34 \pm 1.29 * \#$ \\
RIR+NS & $298.36 \pm 0.36 * \#$ & $25.9 \pm 1.26^{*}+\dagger$ \\
RIR+FSO & $521.87 \pm 0.29^{*} \dagger$ & \\
\hline
\end{tabular}

All values are demonstrated as Mean \pm SEE. ${ }^{*} \mathrm{p}<0.05$ compared to Co+NS; ${ }^{*} \mathrm{p}<0.05$ compared to $\mathrm{Sh}+\mathrm{NS}$. $\dagger \mathrm{p}<0.05$ compared to RIR+NS. SOD: superoxide dismutase, MDA: Malondialdehyde, Co+NS: normal group without ischemia and with oral Normal saline (NS), Sh+NS: sham group subjected to surgery procedure without ischemia induction and with oral NS; IRI+NS: Ischemia induction group with oral NS, IRI+FSO: Ischemia induction group with oral FSO.

Effects of FSO on renal histological damages: No histological damages were observed in the Co+NS and $\mathrm{Sh}+\mathrm{NS}$ groups. In the cortex of the IRI+NS group, a significant increase in the tubular injuries and widespread degeneration of the tubular architecture were observed $(\mathrm{P}<0.05$, Figure 1). However, the intensity of tubular damage decreased in IRI+FSO group compared with $\mathrm{Co}+\mathrm{NS}$ and $\mathrm{Sh}+\mathrm{NS}$ groups $(\mathrm{P}<0.05$, Figure 1$)$.

\section{Discussion}

In this study, occlusion of renal pedicle was used to induce renal IR injury. Renal function was disturbed by increasing the creatinine and BUN levels of serum. The histopathological studies showed high incidence of tubular necrosis in the cortex of rats with renal IR injury.
RIR injury which is common cause of ARFs is often related to high rate of morbidity and mortality among several diseases (17). This condition is characterized by major changes in the renal architecture and functions such as glomerular alteration and filtration reduction, cellular necrosis and tubular damage, tubular leakage and obstruction $(18,19)$. Multiple mediators are involved in the pathogenesis of ARFs such as ROSs, cytokines and adhesion molecules/chemokines which activate the leukocytes and endothelial cells that lead to inflammation, tubular damage and endothelial dysfunction $(20,21)$ and paradoxically subsequent blood reflow enhances the damage in the renal tissue (22). 

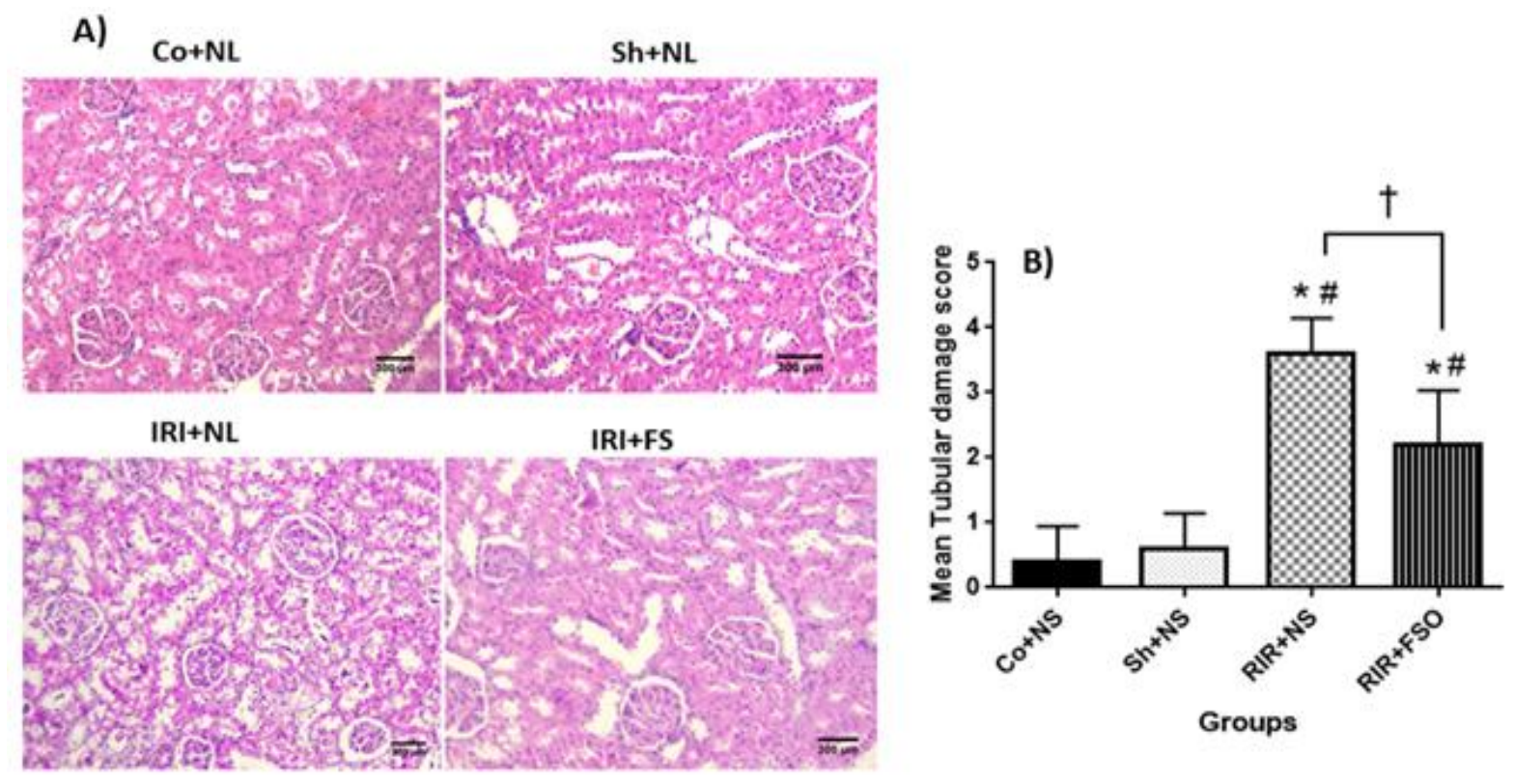

Figure 1. Effect of FSO on tubulo-interstitial damage score in rats with renal IR. A) sections from kidneys showing tubular necrosis in IRI+NS group (more than 70\%) and in IRI+RSO group (about 40\%), H\&E staining $(\times 100)$, B) Comparing the tubulo-interstitial damage score in different groups. ${ }^{*} \mathrm{p}<0.05$ compared to $\mathrm{Co}+\mathrm{NS}$; ${ }^{\#} \mathrm{p}<0.05$ compared to $\mathrm{Sh}+\mathrm{NS} . \dagger \mathrm{p}<0.05$ compared to RIR+NS. BUN: Blood urea nitrogen, Cr: Creatinine, Co+NS: normal group without ischemia and with oral Normal saline (NS), Sh+NS: sham group subjected to surgery procedure without ischemia induction and with oral NS; IRI+NS: Ischemia induction group with oral NS, IRI+FSO: Ischemia induction group with oral FSO.

By increasing the ROSs as an important factor in the pathogenesis of ARFs in the ischemic environment, the antioxidants reduce and resulting in oxidative stress and tissue impairment (23). In the current study, ischemia reperfusion resulted in increased MDA level as a lipid peroxidation production and decreased SOD level. These findings confirm the renal tissue changes in the pathological points and the impaired renal function as described before. A major cellular defense against ROSs is provided by SOD and catalase, which together converts superoxide radicals first to $\mathrm{H}_{2} \mathrm{O}_{2}$ and then molecular oxygen and water (24).

Although, the mechanism of renal IR injury has been understood for several decades, no effective therapies are introduced yet (25). This study was conducted to examine the effects of FSO on the damages induced by renal reperfusion injury. The findings of current study showed that the oral consumption of FSO can decrease the histological damages, oxidative stress and modify renal function disturbances following RIR for 24 hours. The decreased serum creatinine and BUN levels showed the improved renal function in this group. The tubular damages demonstrated studies were reduced compared with RIR injured rats in the histological.

Dietary FS has high contents of omega-3 polyunsaturate fatty acid and lignans with antioxidant properties or/and radical scavenging activities (11). Moreover, FS antioxidant activities were reported under oxidative stress due to diabetes, carbon tetrachloride, and in in-vitro models (26, 27). A possible mechanism for lignans of FS is described by Hosseinian et al. (2007). According to their results, this lignans can produce a stable radical by abstracting a hydrogen atom from the lignan phenol hydroxyl (28).

In the current study, oral administration of FSO in rats with RIR injury reduced MDA content decreased and increased SOD levels in renal tissue. MDA level measurements are widely used as an indicator of lipid peroxidation. In some 
adolescent patients with varicocele, the MDA level increases (29).

Lee et al. (2008) showed the dietary FS $10 \%$ could enhance the antioxidant defenses and decreasing level of MDA in mice underwent pulmonary ischemia reperfusion (21). In Kinniry et al study (2006), the same effects were also observed through reducing the inflammation and oxidative tissue damage in the lung tissue due to acute injury in mice (27).

In a study conducted by Rizwan et al. (2014), the protective effects of dietary FSO on arsenic-induced oxidative damage and nephrotoxicity in rat were investigated. FSO could increase the activities of metabolic enzymes and

\section{References}

1. Simmons MN, Hillyer SP, Lee BH, Fergany AF, Kaouk J, Campbell SC. Functional recovery after partial nephrectomy: effects of volume loss and ischemic injury. $\mathrm{J}$ Urol. 2012;187(5):1667-73.

2. Simmons MN, Schreiber MJ, Gill IS. Surgical renal ischemia: a contemporary overview. J Urol. 2008;180(1):19-30.

3. Himmelfarb J, Joannidis M, Molitoris B, Schietz M, Okusa MD, Warnock D, et al. Evaluation and initial management of acute kidney injury. Clin J Am Soc Nephrol. 2008;3(4):962-7.

4. Carden DL, Granger DN. Pathophysiology of ischaemiareperfusion injury. The Journal of pathology. 2000;190(3):255-66.

5. Masztalerz M, Włodarczyk Z, Czuczejko J, Słupski M, Kedziora J, editors. Superoxide anion as a marker of ischemia-reperfusion injury of the transplanted kidney. Transplantation proceedings. Transplant Proc. 2006 Jan-Feb;38(1):46-8. antioxidant defense system in the arsenic induced oxidative damages. Moreover, the histopathological studies confirmed the biochemical findings showing decrease the tubular necrosis (30).

\section{Conclusions}

On an overall basis, it can be concluded that the oral consumption of FSO for 14 days can protect kidneys against disturbances due to ischemia. Noticing the known antioxidant properties of FSO, it is likely that it induce its effects through antioxidative properties or other unknown pathways which require to be examined by further studies.

6. Chatterjee PK. Novel pharmacological approaches to the treatment of renal ischemia-reperfusion injury: a comprehensive review. Naunyn Schmiedebergs Arch Pharmacol. 2007;376(1-2):1-43.

7. Yamakura F, Taka H, Fujimura T, Murayama K. Inactivation of human manganese-superoxide dismutase by peroxynitrite is caused by exclusive nitration of tyrosine 34 to 3nitrotyrosine. J Biol Chem. 1998;273(23):14085-9.

8. Kaçmaz A, Polat A, User Y, Tilki M, Özkan S, Şener G. Octreotide improves reperfusion-induced oxidative injury in acute abdominal hypertension in rats. J Gastrointest Surg. 2004;8(1):113-9.

9. Glantzounis GK, Salacinski HJ, Yang W, Davidson BR, Seifalian AM. The contemporary role of antioxidant therapy in attenuating liver ischemia-reperfusion injury: A review. Liver Transplantation. 2005;11(9):1031-47.

10. Manna P, Sinha M, Sil PC. Arsenicinduced oxidative myocardial injury: 
protective role of arjunolic acid. Arch Toxicol. 2008;82(3):137-49.

11. Lee JC, Krochak R, Blouin A, Kanterakis S, Chatterjee S, Arguiri E, et al. Dietary flaxseed prevents radiation-induced oxidative lung damage, inflammation and fibrosis in a mouse model of thoracic radiation injury. Cancer Biol Ther. 2009;8(1):47-53.

12. Rizwan S, Naqshbandi A, Farooqui Z, Khan AA, Khan F. Protective effect of dietary flaxseed oil on arsenic-induced nephrotoxicity and oxidative damage in rat kidney. Food Chem Toxicol. 2014;68:99-107.

13. Kitts D, Yuan Y, Wijewickreme A, Thompson L. Antioxidant activity of the flaxseed lignan secoisolariciresinol diglycoside and its mammalian lignan metabolites enterodiol and enterolactone. Mol Cell Biochem. 1999;202(1-2):91-100.

14. Khundmiri SJ, Asghar M, Banday AA, Khan F, Salim S, Levi M, et al. Effect of ischemia reperfusion on sodiumdependent phosphate transport in renal brush border membranes. Biochim Biophys Acta.2005;1716(1):19-28.

15. Kaya H, Sezik M, Ozkaya O, Dittrich R, Siebzehnrubl E, Wildt L. Lipid peroxidation at various estradiol concentrations in human circulation during ovarian stimulation with exogenous gonadotropins. Horm Metab Res. 2004;36(10):693-5.

16. Misra HP, Fridovich I. The role of superoxide anion in the autoxidation of epinephrine and a simple assay for superoxide dismutase. J Biol Chem.1972;247(10):3170-5.

17. Solez K, Kramer EC, Fox JA, Heptinstall RH. Medullary plasma flow and intravascular leukocyte accumulation in acute renal failure. Kidney international. 1974;6(1):24-37.

18. Aragno M, Cutrin JC, Mastrocola R, Perrelli M-G, Restivo F, Poli G, et al. Oxidative stress and kidney dysfunction due to ischemia/reperfusion in rat: attenuation by dehydroepiandrosterone. Kidney Int. 2003;64(3):836-43.

19. Barnes J, Osgood R, Reineck H, Stein J. Glomerular alterations in an ischemic model of acute renal failure. Lab Invest. 1981;45(4):378.

20. Donohoe JF, Venkatachalam MA, Bernard DB, Levinsky NG. Tubular leakage and obstruction after renal ischemia: structural-functional correlations. Kidney Int. 1978;13(3):208-22.

21. Bonventre JV, Zuk A. Ischemic acute renal failure: An inflammatory disease\&quest. Kidney Int. 2004;66(2):480-5.

22. Yano T, Nozaki Y, Kinoshita K, Hino S, Hirooka Y, Niki K, et al. The pathological role of IL-18R $\alpha$ in renal ischemia/reperfusion injury. Lab Inves. 2015;95(1):78-91.

23. Baker GL, Corry RJ, Autor A. Oxygen free radical induced damage in kidneys subjected to warm ischemia and reperfusion. Protective effect of superoxide dismutase. Ann Surg. 1985;202(5):628.

24. Qiao X, Li R-S, Li H, Zhu G-Z, Huang X-G, Shao S, et al. Intermedin protects against renal ischemia-reperfusion injury by inhibition of oxidative stress. Am J Physiol Renal Physiol.2013;304(1):F112-F9.

25. Valko M, Morris H, Cronin M. Metals, toxicity and oxidative stress. Current Med Chem. 2005;12(10):1161-208.

26. Shokeir AA, Hussein AM, Awadalla A, Samy A, Abdelaziz A, Khater S, et al. Protection against renal ischaemia/reperfusion injury: A comparative experimental study of the effect of ischaemic preconditioning vs. postconditioning. Arab $\mathrm{J}$ of Urol. 2012;10(4):418-24.

27. Kinniry P, Amrani Y, Vachani A, Solomides CC, Arguiri E, Workman A, et al. Dietary flaxseed supplementation ameliorates inflammation and oxidative tissue damage in 
experimental models of acute lung injury in mice. J Nutr.. 2006;136(6):1545-51.

28. Lee JC, Bhora F, Sun J, Cheng G, Arguiri E, Solomides CC, et al. Dietary flaxseed enhances antioxidant defenses and is protective in a mouse model of lung ischemia-reperfusion injury. Am J Physiol Lung Cell Mol Physiol. 2008;294(2):L255-L65.
29. Hosseinian FS, Muir AD, Westcott ND, Krol ES. AAPH-mediated antioxidant reactions of secoisolariciresinol and SDG. Org Biomol Chem. 2007;5(4):644-54.

30. Agarwal A, Sharma RK, Desai NR, Prabakaran S, Tavares A, Sabanegh E. Role of oxidative stress in pathogenesis of varicocele and infertility. Urology. 2009;73(3):461-9. 